\title{
Visual hemolysis Index for routine clinical chemistry laboratory parameters
}

\author{
Mohd. Azam Hyder ${ }^{1, *}$, Marghoob Hasan², Abdel Marouf Moheildien ${ }^{3}$ \\ ${ }^{\mathbf{1}}$ Assistant Professor Dept. of Biochemistry, Bhasker Medical College and General Hospital Hyderabad, Telangana, \\ ${ }^{2}$ Lecturer, ${ }^{3}$ Associate Professor, Dept. of Medical Laboratories, College of Applied Medical Sciences, Qassim University, \\ Kingdom of Saudi Arabia
}

*Corresponding Author:

Email: azamhyder@gmail.com

Received: $24^{\text {th }}$ September, 2017

Accepted: $08^{\text {th }}$ January, 2018

\begin{abstract}
Introduction: Interference substances can be significance source of error in the clinical laboratory measurements. Such errors may, in some cases represents hazards in patients. Hemolysis interferes with the Clinical Chemistry Analysis, which influence results of analytes and its leads to erroneous clinical interpretations. Hemolysis of Red cells is inevitable during the collection and handling of blood samples

In usual practice it is very difficult task to perform the rigorous analysis to evaluate interference in routine laboratories. Our objective was to assess effect of Hemolysis on routine biochemistry analysis

Materials and Methods: The study followed the CLSI EP7-A2 protocol, sample were collected from 5 male subjects, aged 2535 years, [0\% (normal pool serum), serial dilution of different hemolysis grades $\left[25 \%\left(1^{+}\right), 50 \%\left(2^{+}\right), 75 \%\left(3^{+}\right)\right.$and $\left.100 \%\left(4^{+}\right)\right]$of sample were prepared by spiking pooled serum with red cell lysate. The interference was studied up to concentrations of $0.5 \mathrm{gm} / \mathrm{dl}$ for hemoglobin. Hemolysis indices graded on the basis of the appearance of the sample qualitatively ranked from "no visible hemolysis" to "4+ hemolysis. Aliquots were analyzed in triplicates using Beckman coulter analyzer. The data was analyzed and $\pm 5 \%$ difference from interferent free sample was taken as acceptable criteria.

Results: The experimental design for laboratory method validation for hemolysis showed that Hemolysed samples starting from $1+$ of hemolysis grade will be rejected for laboratory analysis for clinical chemistry parameters like Total bilirubin, Aspartate amino transferase, Alkaline Phosphatase, Gamaglutamyle transferase, Uric acid and serum potassium. While serum Creatinine hemolysed sample with 4+ hemolysis will not be accepted.

Conclusions: Study results are useful for rejecting / to comment about the potentially misleading results in clinical chemistry parameters of clinical laboratory
\end{abstract}

Keywords: Interferences, Hemolysis, Clinical laboratory analysis, Clinical chemistry.

\section{Introduction}

Chemical interference can be defined as deviation from the result expected for a given concentration of analyte, caused by the presence of another chemical species in the sample. ${ }^{1}$ The presence of interfering substances alters the correct value of the measured result and may lead to inappropriate clinical intervention and compromise patient outcome. ${ }^{2}$ Identification of interference is essential during pre-analytical phase of laboratory analysis. Pre-analytical variables account for $32-75 \%$ of laboratory errors. ${ }^{3}$ Endogenous interferents include the effects of hemolysis, bilirubinemia, lipemia, and paraproteinemia. ${ }^{4}$

Chawla et $\mathrm{al}^{5}$ reported that hemolysis $(0.7 \%)$ was the most common anomaly observed during the assessment of pre-analytical indicators. Moreover, hemolysis account $12.4 \%$ from Emergency Dept. vs. $1.6 \%$ from floor ${ }^{6}$ around the vast majority of hemolysed samples $(>95 \%)$ are attributable to in vitro processes resulting from incorrect sampling procedures or transport. $^{7}$ Hemolysed specimens, the most common reason for rejection, account for $\sim 60 \%$ of rejected specimens, ${ }^{8}$ which increases the turnaround time (TAT).

In the progressing Era of Quality management system in clinical laboratories and patient safety issue, it is highest recommendation that there must be a mechanism to notify clinical personal responsible for patients' care; if the treating physician desire the results, then the laboratory must note the condition of the sample on the report. Some or all tests may not be analytically valid on such a specimen. ${ }^{9}$ Modern clinical chemistry analyzers are equipped with automated systems for detection of lipemia, icteric, and hemolysed samples. With continuous technological development and laboratory automation, a significant reduction in laboratory error scan is expected. It is also reported that only $38 \%$ of laboratories were using automated serum indices for assessment of interference. ${ }^{10}$

However, in laboratories that do not use automated systems for detection and management of pre-analytical interferences, unsuitable samples are detected by means of visual inspection by individual laboratory personnel. Moreover, it is claimed that Visual inspection is not only time consuming, but also highly subjective, nonstandardized and may be a potential source of error ${ }^{11}$. However still this most acceptable practice among the laboratories in absence of any evaluated or standardized hemolysis scale or index for sample rejection.

Hemolysis affects more analytes than does any other type of interference. ${ }^{12}$ In usual practice it is very difficult task to perform the rigorous analysis to evaluate 
interference in routine laboratories. Usually they accept either the manufacture claim or refer the standard text books. It is also claimed that only $8 \%$ of the laboratory performs the in house method validation, while around $95 \%$ follow the manufacturer claims, the data provided is sometimes questionable. ${ }^{13}$ But in the current scenario, to full fill the requirements of accreditation bodies like college of American Pathologist (CAP) or ISO 15189 for the good laboratory practice, it is recommended to perform the method validation which is a tool just to detect error. By method validation at the laboratory site it gives the accurate decision making tool for the laboratory results. ${ }^{14}$

As described by westgard it's important to demonstrate that the method performs well under the operating conditions of every laboratory and that it provides reliable test results for their patients in the light of various variables influencing your system starting from reagent to sample etc. ${ }^{15}$

In the present study our objective was to assess the effect of hemolysis on routine biochemistry analysis and to proposed a visual standard for hemolysed sample compatible to laboratory instrument based on recommended guidelines, which can be easily adopted in routine practice by the laboratory especially which do not have any automated system with capability of performing serum indices.

Design and method: The experimental plan was based on Clinical laboratory standard Institute, USA, CLSI EP7-A2 protocol. ${ }^{16}$

Preparation of hemolysate: Blood collected in EDTA was centrifuged and plasma was removed as much possible., red cells were washed three times with normal saline, then $1 \mathrm{ml}$ Dist $\mathrm{H}_{2} \mathrm{O}$ was added, vigorously mixed on High speed vortex mixer and centrifuged, kept at $20^{\circ} \mathrm{C}$ for one hour, it was thawed at Room temperature and re-centrifuged, supernatant was analyzed for
Hemoglobin on Beckman coulter hematology analyzer, final concentration was adjusted to $0.5 \mathrm{gm} / \mathrm{dl}$ of Hemoglobin with pooled serum,

Samples for Pool serum (PS): 5 Healthy subjects were chosen, aged between 25-35 years. Blood samples were collected as per the CLSI document (GP41-A6Procedures for the Collection of Diagnostic Blood Specimens by Venipuncture; Approved Standard-Sixth Edition) ${ }^{17}$. Venous blood sample collected from each subject after verbal informed consent in BD plain vacutainer by experienced Phlebotomist. Samples were kept for 30 minutes at room temperature for proper clotting. After centrifugation at $3000 \mathrm{rpm}$ for $15 \mathrm{~min}$, pooled serum was prepared and properly mixed. $5 \mathrm{ml}$ kept as interferent free (no hemolysis) and rest $5 \mathrm{ml}$ were spiked with red cell hemolysate with $0.5 \mathrm{gm} / \mathrm{dl}$ in different proportion to get $0.125 \mathrm{gm} / \mathrm{dl}(25 \%), 0.250$ $\mathrm{gm} / \mathrm{dl}(50 \%)$, and $0.375 \mathrm{gm} / \mathrm{dl}(75 \%)$. Neat pooled serum as $0.0 \mathrm{gm} / \mathrm{dl} \quad(0.0 \%)$ and hemolysate $0.5 \mathrm{gm} / \mathrm{dl}$ $(100 \%)$.Whole series consisted of samples with $0 \%$, $25 \%, 50 \%, 75 \%$ and $100 \%$ of the maximal concentration of interferent. Hemolysis indices graded on the basis of the appearance of the sample qualitatively ranked by experienced laboratory technologists from "no visible hemolysis" to "4+ hemolysis," and quantitatively on the basis of \% interferent concentration as $25 \%$ as $1+, 50 \%$ as $2+, 75 \%$ as $3+$, and $100 \%$ as $4+$ (Table 1 ) to match Visual and quantitative decision for hemolysis index( figure 1). Aliquots were analyzed in triplicates using Beckman coulter analyzer.

\section{Formula}

Mean differences $=$ Mean of interferent free sample mean of hemolysed sample

$\%$ mean differences $=$ mean differences $\mathrm{x} 100 /$ mean of interferent free sample

Table 1: Preparation of Test sample scheme

\begin{tabular}{|l|l|c|c|}
\hline Sample & Preparation & $\begin{array}{c}\text { Concentration } \\
(\mathbf{g m} / \mathbf{d l})\end{array}$ & $\begin{array}{c}\text { Concentration } \\
(\mathbf{\%})\end{array}$ \\
\hline normal & Neat pool serum (NP) & 0 & 0 \\
\hline $1+$ & 3 part NP+ 1part NSP & 0.125 & 25 \\
\hline $2+$ & 2 part NP+ 2part NSP & 0.250 & 50 \\
\hline $3+$ & 3 part NP+ 1part NSP & 0.375 & 75 \\
\hline $4+$ & Neat spike pool( NSP) & 0.5 & 100 \\
\hline
\end{tabular}

Fig. 1: Proposed visual hemolysis Index

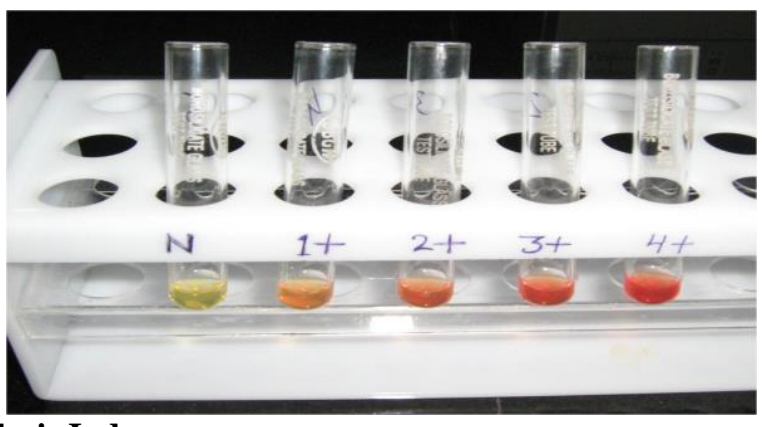




\section{Data analysis}

The data were analyzed using EXCEL 2007; mean differences between interferent free sample (no visual hemolysed) sample to different grades of hemolysed samples and \% of mean of no visual hemolysis were calculated. $\pm 5 \%$ difference from interferent free sample was taken as acceptable criteria.

\section{Results}

The experimental design for laboratory method validation for hemolysis showed that hemolysed samples starting from $1+$ of hemolysis grade will be rejected for laboratory analysis for clinical chemistry parameters like serum Total bilirubin, Aspartate amino transferase, Alkaline Phosphatase, gamma-glutamyl transferase, Uric acid and serum potassium. While serum Creatinine hemolysed sample with $4+$ hemolysis will not be accepted, in other performed clinical chemistry parameters are not significantly affected by different grades of hemolysis as per chosen criteria.

Table 2: Result expressed in \% differences of values from neat pool to different grade of spiked samples

\begin{tabular}{|l|l|c|c|c|c|}
\hline S.no & Analytes & \multicolumn{4}{|c|}{ \% differences from neat pool } \\
\hline & & $4+$ & $3+$ & $2+$ & $1+$ \\
\hline 1 & Glucose & 2.53 & 1.9 & 1.27 & 0.95 \\
\hline 2 & Cholesterol & 4.86 & 3.5 & 2.33 & 0.97 \\
\hline 3 & Calcium & 3.75 & 1.71 & 0.7 & 0.34 \\
\hline 4 & Bili-Total & 17.01 & 13.4 & 12.89 & 6.7 \\
\hline 5 & ALT & 3.89 & 3.33 & 1.67 & 0.56 \\
\hline 6 & AST & 23.48 & 14.78 & 12.17 & 6.09 \\
\hline 7 & ALP & 7.31 & 5.32 & 2.99 & 1.66 \\
\hline 8 & GGT & -65 & -58.33 & -43.33 & -20 \\
\hline 9 & Total Protein & 3.36 & 2.1 & 0.42 & 0 \\
\hline 10 & Albumin & 3.79 & 3.03 & 2.27 & 0.76 \\
\hline 11 & Creatinine & 13.33 & 0 & 0 & 0 \\
\hline 12 & urea & 4 & 1.33 & 1.33 & 1.33 \\
\hline 13 & Uric acid & 37.13 & 11.38 & 8.38 & 2.99 \\
\hline 14 & Sodium & 4.5 & 3.08 & 1.9 & 0.71 \\
\hline 15 & Potassium & 8.27 & 6.77 & 5.26 & 1.5 \\
\hline 16 & Chloride & 4.3 & 2.79 & 1.86 & 0.62 \\
\hline
\end{tabular}

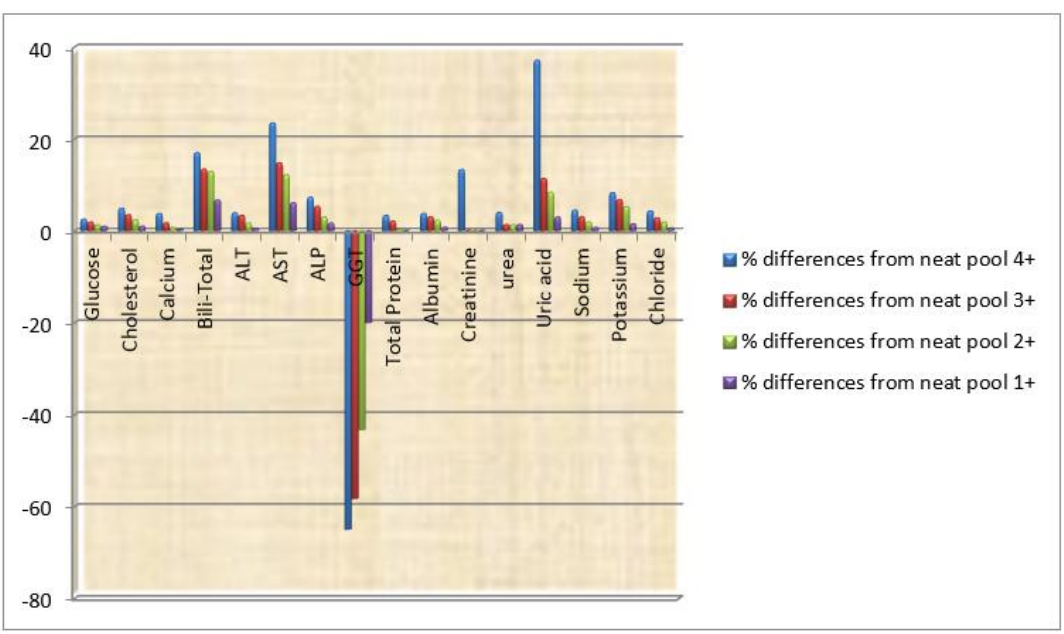

Graph 1: Graphical representation of the \% differences of values from neat pool

\section{Discussion}

The laboratory service plays a key role in patient care. ${ }^{18}$ Laboratory data are estimated to affect $60-70 \%$ of the most important decisions on admission, discharge, and medication. Consequently; laboratory testing is an important source of medical errors affecting patient safety. The majority of the errors in the total testing process originate in the pre-analytical phase. ${ }^{19}$ In recent 
years, there has been increasing interest in quality improvement and patient safety activities in healthcare. Accreditation bodies such as the Joint Commission International (JCI) and the College of American Pathologists (CAP) now require clear and effective procedures for detecting error. ${ }^{11}$ Hemolysis is an important interference factor, must be considered a major pre-analytical variable for the clinical laboratory analysis. ${ }^{12}$ It may occur during the blood collection and sample processing or during transportation. Apart from a few analytes, there are conflicting data in scientific literature on the effect of hemolysis on a wide range of analytes. The discrepancy is related not only to differences in methods for the same analyte, but also to differences in instrumentation. ${ }^{20}$ While to some extent instruments that can subtract or blank the hemoglobin interference by taking spectrophotometric measurements at the wavelength of light where hemoglobin absorbs, in addition to the wavelength at which the analyte absorbs (bichromatic measurements), can minimize the interference, the hemoglobin interference is not eliminated totally, especially when the reagent system used is affected by hemoglobin. ${ }^{13}$ The altered results may lead to repeat tests, incorrect interpretation, wrong diagnosis, and potentially inappropriate intervention and unfavorable outcome for the patients. ${ }^{14}$

The visual evidence of hemolysis is also present in the literatures when the hemoglobin concentration exceeds $20 \mathrm{mg} / \mathrm{dl}^{15,21-23}$ It has been reported that the appearance of serum when $0.1 \%$ of the RBCs are lysed was virtually identical to that of non hemolysed serum and, consequently, would go undetected by laboratory personnel looking for hemolysed specimens. ${ }^{14}$ However, the appearance of serum when $1.0 \%$ of RBCs were lysed was clear and cherry red. Such a specimen would be characterized as having moderate hemolysis. Hemolysis of $1 \%$ of the RBCs affected the measurement of LDH, potassium, AST, and $\mathrm{ALT}^{24}$ with the growing Era of knowledge and advancement in Laboratory medicine to achieve the Goal of clinical laboratory, we should have a clear cut instruction to laboratory staff, about to handle the pre analytical issue, it is also far most expected for all types of labs to have automation with inbuilt facility to handle common preanalytical factors. This must be done especially when the laboratory does not have automation system to assess the grade of hemolysis. It also emphasis the familiarity of phlebotomist pertaining to apply the correct procedure of sample collection, sample accessioning staff regarding the centrifugation speed, aliquot making, and bench technologist regarding the overall influences of hemolysis on Clinical chemistry parameters.

In the present study $\pm 5 \%$ difference from interferent free sample was taken as acceptable criteria, as we practiced the coefficient of variation $(\mathrm{CV} \%)$ for the tested analytes $1 / 3$ of Clinical laboratory improvement amendment proficiency Test (CLIA PT) limits, while other study took $10 \%$ as acceptable limits ${ }^{25}$. In our experiment we found that Hemolysed samples are not accepted for TBIL, AST, ALP, GGT, Uric acid and potassium analyte. Creatinine- Grade 4 Hemolysis will not be accepted. (Table 2),

\section{Conclusion}

Each laboratory should verify the manufacturer claim and there should some visual hemolysis index developed by each lab, which may be used by the laboratory staffs for sample rejection or interpretation of reported value for address the quality requirement and patients' safety. Overall the laboratory must implement an effective detection system to identify specimens with clinically important interferents and a specific policy to prevent reporting of inaccurate results.

\section{References}

1. Martin H K, Mark R, David W B, Ronald J E. A Model for Assessing Interference. Clin Chem.1987;33(7):11213.

2. Kazmierczak SC, Catrou PG. Analytical Interference More than Just a Laboratory Problem. Am J Clin Pathol .2000;113:9-11.

3. Magee LS. Preanalytical Variables in the Chemistry Laboratory,LabNotes.2005;15:1.

4. Galteau MM, Siest G. Drug effects in clinical chemistry. Part2: Guidelines for evaluation of analytical interference. J Clin Chem. 1984;22:275-9.

5. Chawla R, Goswami B, Singh B, Chawla A, Gupta VK, Mallika V.Evaluating Laboratory Performance With Quality Indicators .Lab medicine, 2010;41:5.

6. Burns ER, Yoshikawa N.Hemolysis in Serum Samples Drawn by Emergency Department Personnel versus Laboratory Phlebotomists. Laboratory medicine.2002;5:33.

7. Vermeer HJ, Thomassen E, de Jonge N. Automated processing of serum indices used for interference detection by the laboratory information system. Clin Chem 2005;51:244-7.

8. Carraro P, Servidio G, Plebani M. Hemolyzed specimens: a reason for rejection or a clinical challenge?. Clin Chem 2000;46:306-7.

9. Commission on laboratory accreditation, Laboratory Accreditation Program, laboratory general checklist .2007 .

10. Armbruster DA, Overcash DR, Reyes J. Clinical chemistry laboratory automation in the $21^{\text {st }}$ century. Clin Biochem Rev. 2014;35:143-54.

11. Simundic, AM, Nikolac, N, Ivankovic, N, Dragica FerenecRuzic, D, Magdic, B, Kvaternik, M, Topic, E Comparison of visual vs. automated detection of lipemic, icteric and hemolyzed specimens: can we rely on a human eye?ClinChem Lab Med 2009;47:1361-5.

12. Contois JH, Nguyen R-A. Assay interference: a need for increased understanding and testing. LLC, New Gloucester: Sun Diagnostics, LLC:Sun Diagnostics; 2012.

13. Dimeski G. Interference testing.ClinBiochem Rev.2008;29:1:S43-8.

14. Sciacovelli L, Plebani M. The IFCC working group on laboratory errors and patient safety. Clin Chim Acta 2009;404:79-85. 
15. Westgard $\mathrm{J} \mathrm{O}$, basic method validation, 3rd edition, westgard QC.wisconsin, 2009.

16. McEnroe RJ, Burritt MF, Powers DM, Rheinheimer DW,Wallace BH: Interference Testing in Clinical Chemistry; Approved Guideline-Second Edition. CLSI document EP7-A2. Wayne, PA: CLSI, 2005.

17. ErnstDJ,Balance LO,Calam RR,McCallR, Smith SS,Sazamosi DI,Wrunek DJ :Procedures for the Collection of Diagnostic Blood Specimens by Venipuncture; Approved Standard-Sixth Edition CLSI documents GP41-A6 - Wayne, PA:CLSI, 2007.

18. O'Kane M. The reporting, classification and grading of quality failures in the medical laboratory. Clin Chim Acta 2009;404:28-31.

19. Da Rin G. Pre-analytical workstations: a tool for reducing laboratory errors. Clin Chim Acta 2009;404:6874.

20. MeMn R. Glick, KennethW. Ryder, Analytical Systems Ranked by Freedom from Interferences, clin. chem. 1987;33(8):1453-8.

21. Narayanan S,The Preanalytic Phase, An Important Component of Laboratory Medicine, Am J Clin Pathol 2000;113:429-52.

22. Ji JZ, Meng QH. Evaluation of the interference of hemoglobin, bilirubin, and lipids on Roche Cobas 6000 assays. Clin Chim Acta. 2011;412(17-18):1550-53.

23. Glick MR, Ryder KW, Jackson SA. Graphical comparisons of interferences in clinical chemistry instrumentation. Clin Chem. 1986;32:470-75.

24. Koseoglu M, Hur A, Atay A, Cuhadar S. Effects of hemolysis interferences on routine biochemistry parameters Biochem Med , 2011;21:79-85.

25. Hawkins R,Managing the Pre- and Post-analytical Phases of the Total Testing Process Ann Lab Med 2012;32:5-16. 